\title{
REFLEXIONES ACERCA DEL FUTURO DEL DÓLAR NORTEAMERICANO
}

\author{
REFLECTIONS ABOUT THE FUTURE \\ OF THE NORTH AMERICAN DOLLAR
}

EDDY SALGADO DEFRANC

Universidad Tecnológica Equinoccial

Correo electrónico: esalgado@ute.edu.ec
FeCHA DE RECEPCIÓN: 07/11/2016

FeCHA DE ACEPTACIÓN: 16/12/2016

\section{RESUMEN}

El comportamiento de la balanza comercial es un elemento fundamental en la toma de decisiones económicas, financieras y sociales de los países; por lo tanto, se hace necesario tomar en consideración su comportamiento. Es por ello que en el presente estudio se exhiben los resultados obtenidos en la balanza comercial del Ecuador, todo esto con la finalidad de identificar el comportamiento de la balanza comercial en el país en estos últimos cinco ańos e identificar el porcentaje de participación de la balanza comercial respecto del Producto Interno Bruto en el Ecuador y el crecimiento tanto del PIB como de la balanza comercial.

Palabras clave: Bretton Woods, Richard Nixon, Charles de Gaulle, John Maynar Keynes, moneda de reserva.

\begin{abstract}
In the last months of 1944, World War II was about to end and the United States had become the undisputed world's leading economic power. In this context, what was known today as the Bretton Woods Conference was installed. In this Conference, the International Monetary Fund and the World Bank were created, and the US proposal to convert the dollar into currency world was accepted.

After the signature of the agreement, it is attributed to Charles de Gaulle (future president of France) the phrase that the United States could buy the world with papers. For this not to happen, the United States pledged to exchange their dollars for gold at a rate of \$ 35 a troy ounce. That is, when some Central Bank of the world had hoarded dollars, it could approach the Federal Reserve Bank and exchange these for gold.

However, the US was losing its reserves in gold, reason why in 1971 it only could answer for the $17 \%$ of the dollars that were circulating in the world. In these circumstances, President Richard Nixon, on August 15 of that year, declared the dollar's inconvertibility in gold. Since that date, the dollar has become a fully fiduciary currency (depends on the dollar's confidence) and many authors now agree that the greatest support of the US dollar is actually the US war capacity.
\end{abstract}

Keywords: Bretton Woods, Richard Nixon, Charles de Gaulle, John Maynard Keynes, reserve currency. 


\section{Desarrollo}

Hace tres o cuatro años atrás era frecuente encontrar una gran cantidad de artículos escritos en cualquier parte del planeta que predecían un descalabro del dólar norteamericano. En mayo del 2011 encontramos, por ejemplo, un artículo del diario Oro y Finanzas con un título por demás sugestivo: La devaluación del dólar es inevitable... igual que en 1934. (Oro y Finanzas, 2011). Los títulos de otros artículos eran por igual sorprendentes: El yuan pelea por desplazar al dólar en el comercio mundial. (Rusia Today, 2011); ¿Cuándo llegará el ocaso del imperio del dólar? (Rusia Today, 2012); El yuan amenaza la hegemonía del dólar (Rusia Today, 2013); China y Japón dejarán de usar el dólar en transacciones (Arciniegas, 2013). Cabe aclarar que quienes predecían una segura devaluación del dólar estadounidense estaban pensando en el mediano y largo plazo.

¿Cuál era la razón de semejantes vaticinios sobre el futuro del dólar norteamericano? Pues que entre el 2000 y el 2010 el tipo de cambio del dólar se había reducido en más del $60 \%$ con respecto a las principales divisas mundiales. Durante el período comprendido entre julio de 2001 y julio de 2011 la cotización del euro con respecto al dólar pasó de 0,8477 a 1, 4267. Con relación a los metales preciosos, la devaluación de la divisa norteamericana era aún más acelerada (entre el 2001 y el 2011 una onza de oro aumentó de 270 dólares a más de 1.600).

La crisis europea del 2012 modificó radicalmente el comportamiento del dólar norteamericano. De ese año hasta mediados del 2015, el dólar se ha apreciado con respecto a casi todas las monedas del mundo. Al revisar brevemente la cotización del dólar con respecto a euro vemos que en junio de 2008 un euro se cotizaba en 1.57 dólares; en abril de 2014 la cotización del euro con respecto al dólar había bajado a 1.4; en los primeros meses de 2017 un euro se cotizaba a 1.04 dólares (El Economista, 1917). La apreciación del dólar es más notoria debido a las medidas proteccionistas y de carácter fiscal anunciadas por Donald Trump (El País, 2017). La guerra monetaria entre el dólar y el euro, lo es también entre el dólar y el yuan. Una apreciación del dólar produce una suerte de fuga de capitales en China, lo cual obliga al gobierno chino a tomar medidas para apreciar al yuan (Gestión PE, 2017).

Esta guerra monetaria se presenta cada vez más explícita. Por ejemplo, en mayo del año pasado (2014) Rusia y China firmaron un acuerdo según el cual la empresa rusa Gazprom suministrará a la China 38.000 millones de metros cúbicos de gas anuales durante 30 años. El acuerdo es por unos 400.000 millones de dólares; gigantesco en sí, el acuerdo contempla el que Rusia aceptó que China pague con su propia moneda, es decir en yuanes. Esto quiere decir (y esto es la guerra monetaria $\left.^{1}\right)$ que el yuan de China comienza a posicionarse como moneda de pago internacional, lo cual debilita al dólar de los Estados Unidos. Este acuerdo entre Rusia y China no es el único. "En 2013, la petrolera rusa Rosneft firmó un acuerdo con la empresa China Sinopec para el suministro de 100 millones de toneladas de petróleo en los próximos diez años. El valor del contrato podría alcanzar los 85.000 millones de dólares" (Rusia Today, 2014). Este tipo de acuerdos y contratos proliferan por el mundo entre China y Brasil, China y el Japón, Brasil y la Argentina.

En este contexto, la pregunta que unos y otros se hacen en el mundo tiene que ver con el comportamiento en el mediano y largo del dólar norteamericano. El objetivo de este artículo es ofrecer elementos de análisis respecto al comportamiento futuro del dólar estadounidense. Por futuro habría que entender en los próximos 20 o 30 años.

Para alcanzar una mejor comprensión del presente, debemos recurrir siempre a la historia y en este caso remontarnos a lo sucedido en Bretton Woods (New Hampshire) en 1944. La Segunda Guerra Mundial estaba a punto de llegar a su fin y los Estados Unidos se había convertido en la indiscutida primera potencia económica mundial. Entre otros aspectos, este país había logrado

1 Los países del BRICS estarían buscando posicionar una moneda que los represente a todos. 
Eddy Salgado Defranc

Reflexiones acerca del futuro del dólar norteamericano

acaparar un $75 \%$ del oro mundial, factor fundamental para entender el problema que vamos a estudiar (Triffin, 1982).

En Bretton Woods no sólo que fueron creados el FMI y el Banco Mundial, sino que los países signatarios del Acuerdo aceptaron que el dólar se convierta en moneda de curso mundial, es decir que pueda circular no sólo en los Estados Unidos, sino en todo el planeta. Desde ese momento los países podían, a voluntad, utilizar para saldar sus intercambios (tanto de productos como de servicios) tanto el oro como el dólar. De esta manera, inteligentemente, se obligaba a todos los países del mundo a tener su Reserva Monetaria Internacional (RMI) en dólares (Pino Santos, 1986).

Entre las anécdotas de ese período, cabe recordar que el economista inglés John Maynard Keynes - para muchos el más influyente economista del siglo XX- estuvo en desacuerdo con los mecanismos de reorganización del sistema financiero internacional que se adoptaron en ese pequeño poblado norteamericano. De igual manera, el futuro presidente francés Charles de Gaulle fue particularmente crítico de lo resuelto en New Hampshire (inclusive se le atribuye la frase que desde Bretton Woods los EE.UU se podían comprar el mundo con papeles) (Monteverde, 1989).

Para darle algo de coherencia a los acuerdos de Bretton Woods, los EE.UU se comprometieron a respaldar los dólares que a futuro circularían por el mundo con oro. Efectivamente, cuando un banco central de cualquier parte del mundo hubiese acumulado dólares en su RMI, podía exigir el cambio de esos dólares por oro a razón de 35 dólares la onza troy. Recordemos que antes habíamos indicado que a mediados de la década del 40, EE.UU tenían en sus arcas las $3 / 4$ de las reservas mundiales de oro.
A estas alturas del análisis es necesario aclarar un problema de teoría monetaria poco comprendida, ¿cuál es el respaldo para la emisión monetaria? La respuesta en este caso es unívoca. El verdadero respaldo del dinero en circulación es la producción. Es lógico, si la principal función del dinero es servir de intercambio para la circulación de mercancías, son la sumatoria en valores de estas (expresadas en precios) lo que da respaldo a los billetes en circulación.

El patrón oro, es decir cuando los bancos emitían dinero de acuerdo a la cantidad de oro que tenían en sus bóvedas, fue eliminado en el todo el mundo allá en los primeros ańos de la década del 30 del siglo pasado. Recordemos, que fue Australia el primer país en abandonar el patrón oro en 1930.

Realizada esta aclaración, se debe indicar, entonces, que los dólares que circulan dentro de los EE.UU tienen respaldo en el PIB norteamericano; el problema radica, por lo tanto, en el respaldo para los dólares que circulan fuera de ese país. Son éstos los que necesitan algún respaldo y que según lo acordado en Bretton Woods tenía que ser el oro.

Pasó el tiempo y llego el año de 1958, año en el cual EE.UU tuvo por primera vez un déficit de 3.000 millones en la balanza de pagos. ¿Qué quiere decir esto? Que desde ese año más son las mercancías y servicios que recibe los EE.UU desde el resto del mundo, que los servicios y mercancías que entrega este país al resto del mundo. ¿Cómo paga los EE.UU ese déficit? Lo hace con su propia moneda y ese es el privilegio que recibió en 1944 en New Hampshire. Más adelante, y debido a estos déficit, EE.UU comenzó a perder sus reservas de oro. Los bancos centrales del mundo acumulaban dólares que, según lo acordado, eran trocados por oro. Veamos algunos datos en el cuadro No 1 : 


\section{CuAdro 1. EVOLUCIÓN DE LAS ObLIGACIONES INTERNACIONALES}

Y LAS RESERVAS DE ORO DE LOS Estados Unidos. Miles de MiLlones de DÓlares

\begin{tabular}{l|c|c}
\hline \multicolumn{1}{c}{ Ańo } & $\mathbf{1 9 4 9}$ & $\mathbf{1 9 6 0}$ \\
\hline $\begin{array}{l}\text { Activos en oro y divisas } \\
\text { convertibles }\end{array}$ & 28.1 & 25.9 \\
\hline De esto solo en oro & 24.6 & 18.4 \\
\hline $\begin{array}{l}\text { Total de obligaciones } \\
\text { con el extranjero }\end{array}$ & 8.2 & 23.5 \\
\hline
\end{tabular}

Fuente: R. Triffin: The Internacional Monetary Position of United States, pp. 228-29. En Oscar Pino Santos, La crisis del capitalismo, editorial Nuestro Tiempo, México D.F., primera edición 1982, p. 85.

EE.UU fue perdiendo sus reservas de oro y en cambio se fue incrementando la cantidad de dólares circulando por el mundo (en el cuadro No 1 denominados como "obligaciones con el extranjero"). Según se desprende del mismo cuadro, ya en 1960 la cantidad de dólares circulando por el mundo era mayor que las tenencias de oro por parte de los EE.UU (Aguilar Monteverde, 1993).

El déficit en la cuenta corriente de la balanza de pagos (específicamente en la balanza comercial) nunca se revirtió, de manera que el país del norte fue perdiendo más y más sus tenencias de oro. En el cuadro No 2 tenemos una mejor perspectiva de este proceso:

\section{Cuadro 2. Reservas de oro de los EE.UU.}

EN MILES DE MILLONES DE DÓLARES

\begin{tabular}{c|c}
\hline Años & Reservas \\
\hline 1949 & 24.6 \\
\hline 1959 & 18.4 \\
\hline 1969 & 11.9 \\
\hline & Fuente: Ibídem, p. 87 \\
\hline
\end{tabular}

Las evidencias son incontrastables. Entre 1949 y 1969 los EE.UU habían perdido más del 50\% de sus tenencias de oro, mientras que sus obligaciones con el extranjero seguían creciendo. El año de crisis fue 1971. En ese año sus tenencias de oro habían bajado a 10.510 millones de dólares, mientras que la deuda monetaria externa -dólares en circulación fuera del país- era de 60.000 millones de dólares. En otras palabras, en 1971 los EE.UU sólo podía responder con oro por un $17 \%$ de los dólares que circulaban por el mundo (Castro Ruz, 1989).

RichardNixon, presidentenorteamericanodelaépo$\mathrm{ca}$, resolvió el problema de una manera sui géneris: el 15 de agosto de ese año declaró la inconvertibilidad del dólar en oro, es decir los EE.UU "se negaba en lo sucesivo a convertir los dólares en oro, no obstante los acuerdos de Bretton Woods y a pesar de que para entonces las arcas de los bancos centrales de los otros países estaban repletas hasta el tope de reservas en esa moneda" (Pino Santos, 1986).

Después de 1971 los EE.UU utilizaron con largueza los privilegios adquiridos en Bretton Woods, lo cual se puede advertir con facilidad en el cuadro No3.

\section{CUADRO 3. Posición INTERNACIONAL DEL DÓLAR} (EN MILES DE MILLONES DE DÓLARES)

\begin{tabular}{|c|c|c|}
\hline Años & $\begin{array}{l}\text { Reservas } \\
\text { de oro }\end{array}$ & $\begin{array}{c}\text { Obligaciones en } \\
\text { poder de extranjeros }\end{array}$ \\
\hline 1960 & $17,8(1)$ & 40,9 (3) \\
\hline 1970 & $11,1(1)$ & 97,7 (3) \\
\hline 1980 & $11,2(2)$ & $202,9(4)$ \\
\hline \multicolumn{3}{|c|}{$\begin{array}{r}\text { Fuente: (1) Economic Report of the President, } \\
1969 \text {, USGPO, p. } 330 . \\
\text { ral Reserve Bulletin, noviembre de 1982, p. } 455 . \\
\text { Internacional Economic Report of the President, } \\
1975 \text {, USGPO, p. } 143 . \\
\text { Economic Indicators, diciembre de 1982, p. } 86 . \\
\text { so Aguilar Monteverde, Apuntes sobre economía } \\
\text { al, México D.F., Editorial Nuestro Tiempo, S.A. } \\
\text { 1991, p. } 189 .\end{array}$} \\
\hline
\end{tabular}


En la actualidad el conocimiento de estos datos se ha complicado, debido a que la Reserva Federal el 23 de marzo de 2006 decidió no publicar el dato del M3 (recordemos que en la teoría de los agregados monetarios se utiliza el M1, el M2, el M3, etcétera). Datos aproximados sobre la cantidad de dólares que circulan por el mundo recorren las páginas de Internet y ameritan otro artículo similar al actual.

¿Cómo opera el proceso en términos generales? O mejor dicho ¿Cómo salen los dólares de los EE.UU hacia el resto del mundo? Fácil e ingenua pregunta, pero difícil y delicada respuesta. Se intentará, sin embargo, una breve explicación.

El mare magnum se inicia con el déficit fiscal del gobierno federal. ¿Cómo se paga este déficit? De las dos únicas maneras como se lo puede hacer: con endeudamiento o con emisión inorgánica de dinero. El proceso se inicia en los dos casos mediante la venta de bonos del tesoro norteamericano. Cuando los compradores son particulares o gobiernos extranjeros, el gobierno de los Estados Unidos incrementa sus descomunales niveles de deuda del gobierno federal. En cambio, cuando quien compra esos bonos es el banco de la Reservas Federal, se está incrementando la base monetaria (denominada en los libros de macroeconomía como dinero de alta potencia), es decir se está emitiendo y poniendo en circulación nuevo dinero. Este último caso es lo que se denomina emisión inorgánica de dinero, pues es emisión monetaria sin respaldo en la producción. ¿Por qué esta emisión inorgánica no genera inflación en los EE.UU? Simplemente, porque ese dinero sale al resto del mundo (Dornbusch, 2014).

¿Físicamente en qué momento salen esos dólares de la economía norteamericana? Salen, por ejemplo, cuando desde los EE.UU se pagan las importaciones, que -lo repetimos nuevamente- EE.UU tiene el privilegio de pagarlo con su propia moneda. Salen también cuando ese país paga sus gastos militares en el exterior. ¿Cuánta razón tuvieron J.M. Keynes y Charles de Gaulle para estar en contra de los acuerdos de Bretton Woods? (Cuadrado Roura, 2001).
Así, cómo no recordar las palabras de John Connaly, Secretario del Tesoro del gobierno norteamericano en la época de Nixon, quien, en 1971 dijo "El dólar es nuestra moneda, pero es vuestro problema".

\section{Conclusiones}

1. El mayor triunfo de los Estados Unidos al finalizar la Segunda Guerra Mundial fue lograr que en los acuerdos de Bretton Goods el dólar pase a ocupar el papel del oro en el comercio internacional. A partir de Bretton Goods el activo más importante en la RMI de los diferentes países del mundo dejó de ser el oro, puesto que pasó a ser ocupado por el dólar norteamericano. Por esta razón se popularizó la frase de que el dólar era tan bueno como el oro. Este hecho fue posible debido a que al finalizar la Guerra EE.UU. poseía gran parte del oro mundial. La situación cambió cuando este país perdió gran parte de ese oro debido a su déficit en la balanza de pagos desde finales de la década del siglo pasado. Nixon resolvió el problema simplemente declarando la inconvertibilidad del dólar en oro el 15 de agosto de 1971.

2. En la actualidad circulan por el mundo billones de dólares sin respaldo. Se desconoce la cifra exacta, debido a que EE.UU. decidió no publicar el listado total de los agregados monetarios.

3. El surgimiento de nuevas potencias económicas a partir de los inicios del siglo XXI pone en entredicho el papel de moneda universal del dólar norteamericano. De estas potencias, la más importante es la República Popular China, país que ha logrado acumular reservas internacionales en el orden de los 3 billones 800.000 millones de dólares. Cabe aclarar que en este caso el dólar es la moneda de cuenta, pero eso no quiere decir que la República Popular China tiene esta cantidad en dólares norteamericanos. Estos más de tres billones de dólares, la China lo tiene en diferentes activos en sus reservas internacionales. Este dato da cuenta por sí solo del poderío de la economía de ese país. 
4. El hecho que los países pertenecientes al recién creado grupo de los BRICS hayan decidido realizar sus intercambios comerciales con sus propias monedas, dejando de lado al dólar de los EE.UU., configura lo que hoy se conoce como las Guerras Monetarias. El hecho de que los dólares que circulan por el mundo sean totalmente fiduciarios (es decir su único respaldo es la confianza que la gente tenga en ellos), nos indica la debilidad estructural de la moneda de ese país.

5. Debidoaquevarios paísesestén aceptandoaqueen un futuro cercano sus exportaciones a China sean pagas con yuanes (pusimos como ejemplo el caso de Rusia) hace prever el inicio de una nueva fase de esta Guerra Monetaria. Este tema, por supues to, amerita otro u otros artículos como el actual.

\section{Referencias bibliográficas}

- Aguilar Monteverde, A. (1991). Apuntes sobre economía internacional. México: Nuestro Tiempo, S.A.

- Aguilar Monteverde, A. (1993). Las tres crisis del capitalismo contemporaneo. México: Nuestro Tiempo.

- Arciniegas, R. (2013). China y Japón dejarán de usar el dólar en transacciones. Perspectiva latinoamericana, 14-21.

- Castro Ruz,F.(1989). InformealMovimientodelos no Alineados. La Habana: Cuba Independiente.

- Cuadrado Roura, J. (2001). Politica Económica. Madrid: Mc Graw Hill.

- Dornbusch, R. (2014). Macroeconomía. Mc Graw Hill.

- El Economista. (18 de Enero de 1917). Obtenido de http://www.eleconomista.es/firmas/ noticias/8066993/01/17/Caida-del-euro-osubida-del-dolar.html.

- El País. (17 de enero de 2017). Obtenido de htpp:/cincodias.elpais.com/cincodias/2017/02/ 17/mercados/1487341279_027423.html\# contenedor.

- Gestión PE. (1 de enero de 2017). Obtenido de HTTP://GESTION.PE/ECONOMIA/ CHINA-INTENTA-ECLIPSAR-AL-DOLAR-APRECIACION-YUAN-2178543.
- Monteverde, A. (1989). Apuntes sobre economía internacional. México: Nuestro Tiempo.

- Oro y Finanzas. (25 de Mayo de 2011). La devaluación del dólar es inevitable... igual que en 1934. Oro y Finanzas, págs. 8-10.

- Pino Santos, O. (1986). La crisis del capitalismo. México: Nuestro Tiempo.

- Rusia Today. (26 de febrero de 2011). actualidad /view/12889/yuan peleaporhegemonía. Recuperado el 14 de junio de 2012, de http://www.rt.com

- Rusia Today. (31 de julio de 2012). Rusia Today. Recuperado el 17 de febrero de 2013, de http:www.actualidad.rt.com/actualidad/viewocaso-imperio-dólar

- Rusia Today. (26 de enero de 2013). Rusia Today. Recuperado el 14 de marzo de 2013, de http://www.noticias.rt.com/noticias/view/ 25229-yuan-amenaza- hegemonía-dólar

- Rusia Today. (21 de Septiembre de 2014). Rusia Today. Recuperado el 14 de mayo de 2015, de http://actualidad.rt.com/actualidad/ view/140852-union-rusia-china-temor-eeuuguerra fria

- Triffin, R. (1982). The International monetary position of United States. México D.F.: Nuestro Tiempo. 